\title{
Physical Chemistry of the Lipids
}

\section{of Human Atherosclerotic Lesions}

\author{
DEMONSTRATION OF A LESION INTERMEDIATE BETWEEN \\ FATTY STREAKS AND ADVANCED PLAQUES
}

\author{
Saul S. Katz, G. Graham Shipley, and Donald M. Small \\ From the Biophysics Division, Department of Medicine, Boston University \\ School of Medicine, Boston, Massachusetts 02118
}

A в S T R A C T 95 individual human atherosclerotic lesions from 26 persons were classified into three groups under the dissecting microscope: fatty streaks, fibrous plaques, and gruel (atheromatous) plaques. Each lesion was isolated by microdissection, its lipid composition determined by chromatography, and the physical states of the lipids identified by polarizing microscopy and in some cases by X-ray diffraction. The composition of each lesion was plotted on the in vitro phase diagram of the major lipids of plaques: cholesterol, cholesterol ester, and phospholipid. The observed physical states were compared with those predicted by the location of the lipid composition on the phase diagram.

The most severe lesions (gruel plaques) had an average lipid composition of cholesterol $31.5 \pm 1.9 \%$, cholesterol ester $47.2 \pm 2.3 \%$, and phospholipid $15.3 \pm 0.5 \%$. Their compositions fell within the three-phase zone of the phase diagram, predicting the lipids to be separated into a cholesterol crystal phase, a cholesterol ester oily phase and a phospholipid liquid crystalline phase. In addition to the phospholipid liquid crystalline phase of membranes and myelin-like figures demonstrable by electron microscopy, polarizing microscopy revealed the other two predicted phases, isotropic cholesterol esterrich droplets and cholesterol crystals. X-ray diffraction studies verified the identity of the crystals as cholesterol monohydrate.

Fibrous plaques also had an average lipid composition

Presented in part at the Annual Meeting of the American Heart Association, 17-20 November 1975, Anaheim, Calif. 1975. Circulation. 52: II-104. (Abstr.)

Dr. Katz is a recipient of a Medical Research Council of Canada Fellowship.

Received for publication 23 October 1975 and in revised form 16 February 1976. within the three-phase zone of the phase diagram. Polarizing microscopy revealed the presence of cholesterol monohydrate crystals and lipid droplets in all of these lesions; the droplets were predominately isotropic in 28 of the 31 fibrous plaques. Although these lesions had less free cholesterol and more cholesterol ester than gruel plaques, they were otherwise similar.

Fatty streaks had compositions within both the twoand three-phase zones of the phase diagram. Compared with gruel plaques, the fatty streaks within the twophase zone, defined as "ordinary," had more cholesterol ester, less free cholesterol, a higher cholesteryl oleate/ cholesteryl linoleate ratio, a lower sphingomyelin/lecithin ratio, more anisotropic lipid droplets, and rare or no cholesterol crystals. Those lesions within the three-phase zone had many chemical and physical features intermediate between ordinary fatty streaks and gruel plaques. Moreover, $68 \%$ of these "intermediate" lesions had no cholesterol crystals by polarizing microscopy in spite of their compositions being within the three-phase zone, indicating the cholesterol ester oily phase or the phospholipid phase or both were supersaturated with cholesterol. Identification of this group of intermediate lesions provides evidence that some fatty streaks may be precursors of advanced plaques.

\section{INTRODUCTION}

Atherosclerosis is associated with the focal accumulation in arteries of three major classes of lipids: cholesterol, cholesterol ester, and phospholipid. At body temperature, these lipids can exist in at least three distinct physical states-crystalline, liquid crystalline, and liquid-depending largely on their relative concentrations and lipidlipid interactions. For example, cholesterol, which is 
crystalline in an aqueous environment, can be solubilized by phospholipid up to equimolar amounts (1). It can also be incorporated into liquid cholesterol ester to a small extent (2), but if the amount of cholesterol exceeds these limits of solubility, it will be present, under equilibrium conditions, in the form of crystals. Cholesterol esters may be liquid, liquid crystalline, or crystalline at body temperature depending on the relative amounts of individual cholesterol esters and on the amount of solubilized cholesterol or other minor lipids such as triglycerides (2). Finally, phospholipids such as lecithin and sphingomyelin are present in excess water as lamellar liquid crystals $(3,4)$. The relationships between these lipids have been defined in a model system by phase equilibrium experiments with water and chemically pure lipids (5). The results can be expressed in a simplified condensed phase diagram using triangular coordinates (Fig. 1). Zone $I$ is a one-phase region of phospholipid lamellar liquid crystals in which up to $33 \%$ cholesterol and about $2 \%$ cholesterol ester by weight may be incorporated. Zone II is also a single phase but the major component, cholesterol ester, can incorporate only up to about $8 \%$ cholesterol and less than $1 \%$ phospholipid. This phase is liquid at $37^{\circ} \mathrm{C}$ if cholesteryl linoleate is the predominant cholesterol ester, and liquid crystalline if cholesteryl oleate is the predominant ester (D. M. Small, unpublished observations). Since the cholesterol ester-rich phase forms droplets in atherosclerotic lesions, it may be called the lipid droplet phase. Zone III is a two-phase zone consisting of cholesterol ester droplets and phospholipid liquid crystals, and zone IV is a threephase zone with cholesterol monohydrate crystals forming the third phase.

Plaque lipid compositions from previous studies (612) are plotted on the phase diagram in Fig. 2a. They all fall within the three-phase zone, and, therefore, we would expect that these plaques would contain cholesterol crystals, cholesterol ester-rich droplets, and phos-

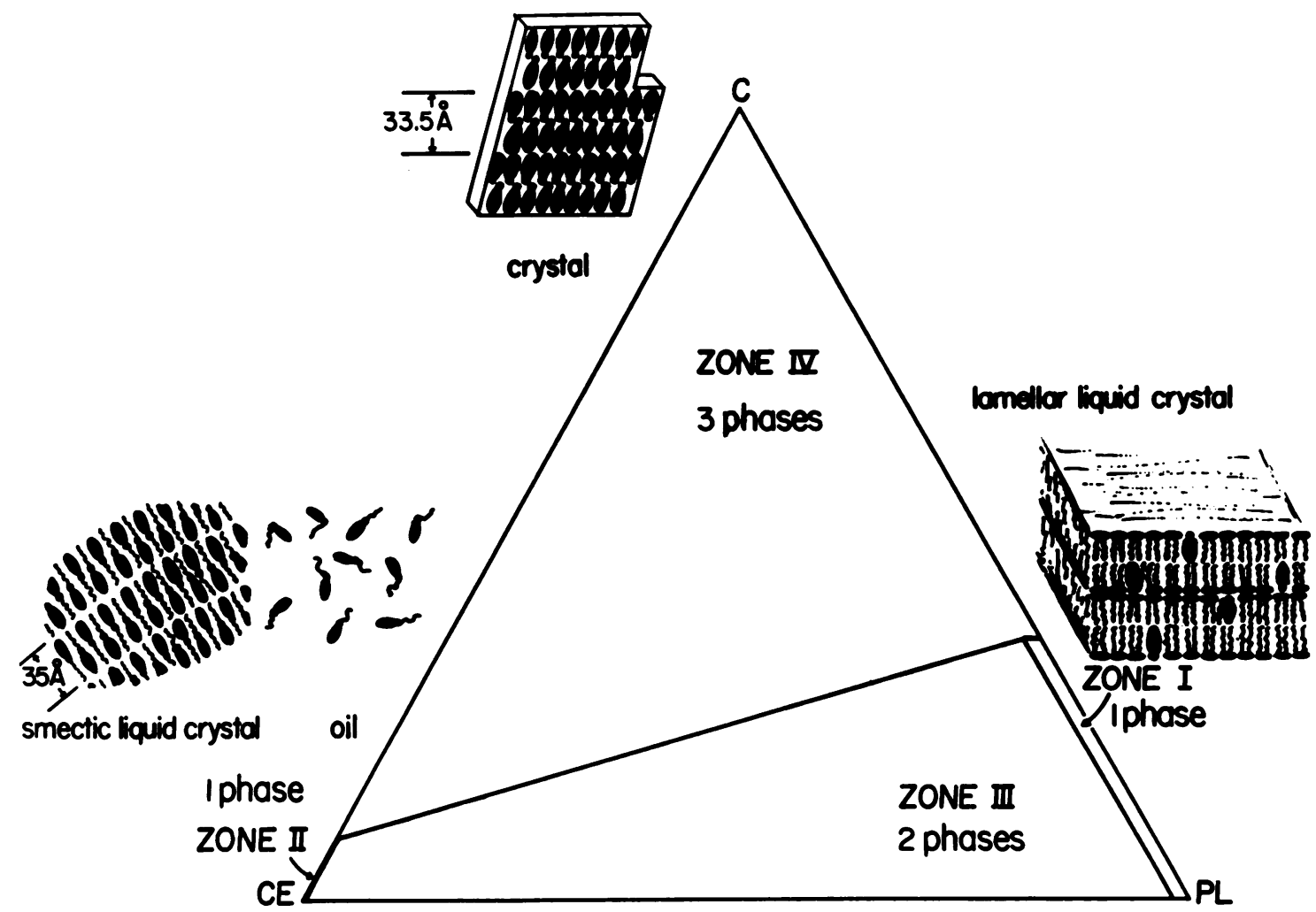

FIgURE 1 Phase diagram of three-component system, cholesterol (C), phospholipid (PL), cholesterol ester $(\mathrm{CE})$, in excess water at $37^{\circ} \mathrm{C}$ and 1 atmosphere pressure (for derivation of phase diagram, see reference 5 ). Zone 1 has a single phase of phospholipid lamellar liquid crystal with up to $33 \%$ cholesterol and $2 \%$ cholesterol ester. Zone II also has a single phase composed of liquid or liquid crystalline cholesterol ester. Mixtures in zone III have both the cholesterol ester and the phospholipid phase present. In zone IV there is a third phase as well, cholesterol monohydrate crystals. Schematic molecular representation of the phases shown near each apex of the triangle. Phospholipid molecule shown as $\leftarrow$, cholesterol - - , and cholesterol ester Molecules of cholesterol ester form isotropic oil droplets when random, and birefringent droplets when ordered in layers $35 \AA$ thick. 


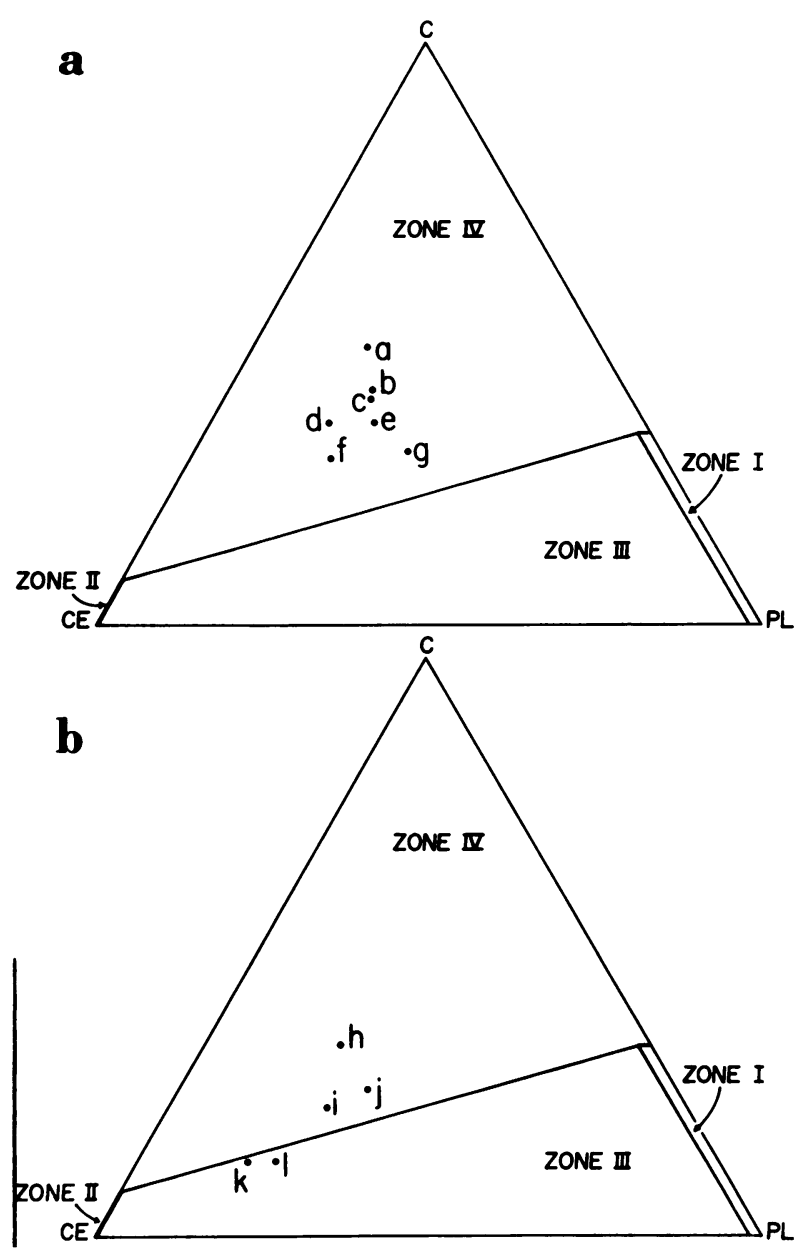

FIGURE 2 (a) Lipid composition of advanced plaques taken from the literature: (a) Luddy et al. (11); (b) Buck and Rossiter (6); (c) Smith and Slater (7); (d) Bottcher and Woodford (12); (e) Field et al. (9); ( $f$ ) Panganamala et al. (8); and $(g)$ Dayton and Hashimoto (10). (b) Lipid composition of fatty streaks taken from the literature: (h) Insull and Bartsch (23), fatty streaks, group II; (i) Insull and Bartsch (23), fatty streaks, group I; (j) Panganamala et al. (8), aortic fatty streaks without foam cells; $(k)$ Panganamala et al. (8), aortic fatty streaks with foam cells; (l) Smith (24).

pholipid liquid crystals, either as cellular membranes or concentric myelin figure-like deposits. Despite the anticipated presence of these lipid states in all such plaques, surprisingly little effort has been made to characterize them or to define their role in the pathogenesis of atherosclerosis. This is due, in part, to the fact that lipid physical states cannot be studied by standard pathological techniques, since routine tissue processing, involving dehydration, fixation, or freezing, changes these states drastically. Treating tissues with organic solvents dissolves the lipids, while freezing before sectioning crystallizes them, precluding appreciation of the lipid states which had existed in vivo. Nevertheless, by means of other techniques, all three phases have been recognized in plaques. Myelin figures and lipid bilayers characteristic of phospholipid liquid crystals have been identified with electron microscopy $(13,14)$. The lipid droplet phase has been seen with polarizing microscopy $(5,15$, 16) and has recently been characterized (17-19). The crystal phase, recognized in histopathologic sections as "cholesterol clefts," has been assumed to be cholesterol ester $(13,20)$, although Bogren and Larsson (21) have suggested it is cholesterol monohydrate on the basis of $\mathrm{X}$-ray diffraction studies of two plaques. Cholesterol crystals have also been identified in plaques by means of polarizing microscopy $(5,22)$.

Fatty streak lipid compositions taken from the literature $(8,23,24)$ fall within both the two- and threephase zones (Fig. 2b). Descriptively, they are all fatty streaks as defined by the World Health Organization (WHO) classification of atherosclerotic lesions (25), but chemically, they may be divided into two groups on the basis of the model phase diagram. Those in zone III do not have excess free cholesterol, and we shall call them "ordinary" fatty streaks, while those in zone IV have excess free cholesterol, and we shall designate them as "intermediate lesions," because they have chemical and physical features intermediate between ordinary fatty streaks and advanced lesions (see below).

In the present study, the chemical composition of 95 individual atherosclerotic lesions was determined by microanalytical techniques. The physical states of the lipids in each lesion were determined by hot-stage polarizing light microscopy; X-ray diffraction studies of 13 lesions confirmed the observed physical states. Chemical and physical findings were evaluated in terms of the phase diagram derived from in vitro experiments. Finally, the physical and chemical features of different types of atherosclerotic lesions were compared, and evidence was found of a continuum from ordinary fatty streaks to advanced necrotic plaques.

\section{METHODS}

\section{Tissue material}

19 necropsy aortas and 7 surgically resected arterial specimens were studied. Patients' ages ranged from 28 to 89 $\mathrm{yr}$, with an average age of 66.0. There were 12 females and 14 males. Aortas were obtained within $24 \mathrm{~h}$ of death, and all physical studies were performed on fresh tissue to avoid artefacts introduced by freezing or fixation. Changes in physical states of lipids induced by cooling to ambient temperature were reversible, and were limited to liquid-toliquid crystalline transitions in some of the droplets. Causes of death were pulmonary embolism $(n=2)$, carcinoma $(n=$ $10)$, congestive heart failure $(n=3)$, subacute bacterial endocarditis $(n=1)$, cerebrovascular accident $(n=2)$, and myocardial infarction $(n=1)$. The surgical specimens were from abdominal aortic aneurysmectomy $(n=2)$ and iliofemoral endarterectomy or bypass $(n=5)$. Thin-layer 
chromatography of the lipids of both surgical and autopsy specimens showed no more than trace amounts of free fatty acids and less than $2 \%$ of lysolecithin, confirming that there was no major postmortem autolysis of lipids. Furthermore, comparable lesions in the surgical and autopsy groups were similar by microscopy and X-ray diffraction as well as by chemical analysis, indicating that physical states of lipids in lesions from the two sources were similar, and therefore surgical and autopsy specimens will not be considered separately.

Aortas were opened longitudinally, blood was washed free from the luminal surface, and the adventitia was then removed. Lesions were examined under a dissecting microscope with a magnification $\times 10$ to $\times 40$, and were classified according to WHO guidelines (25) into fatty streaks, fibrous plaques, gruel (atheroma) plaques, and complicated plaques. Fatty streaks were superficial, flat, or slightly raised yellow intimal lesions, often consisting of clusters of small yellow dots. Fibrous plaques were raised firm lesions with a gray fibrous cap overlying a yellow lipid-rich center. Gruel plaques were similar, but the core was necrotic ("an atherosclerotic plaque in which fatty softening is predominant". reference 25). Clusters of crystals were often visible to the unaided eye within the gruel material. Complicated plaques with hemorrhage, calcification. ulceration, or overlying thrombus were not included in the present study. Individual lesions were microdissected with a scalpel and fine forceps, and minced on a glass slide. Portions were taken for chemical analysis, $\mathrm{X}$-ray diffraction, and polarizing microscopy.

\section{Chemical methods}

Thin-layer chromatography. Lipids from minces or homogenates of plaques were extracted overnight in $10 \mathrm{vol}$ of chloroform-methanol (2:1, vol/vol), after which a Folch procedure (26) was carried out. Since the total amount of lipids in each individual lesion was often less than a milligram, it was necessary to use sensitive microanalytical techniques to separate and quantitate the extracted lipids. Quantitative thin-layer chromatography (27) was used to measure the free cholesterol, fatty acid, triglyceride, cholesterol ester, lysolecithin, sphingomyelin, and lecithin. Pyrex plates, $20 \times 20 \mathrm{~cm}$, with $0.25 \mathrm{~mm}$ silica gel $\mathrm{G}$, were divided into 30 lanes, $6 \mathrm{~mm}$ wide. After washing overnight in diethyl ether, the plate was activated for $30 \mathrm{~min}$ at $120^{\circ} \mathrm{C}$, and samples applied in duplicate on the right and left halves of the plate. A standard solution of cholesterol (Nu-Chek Prep, Elysian, Minn.), triglyceride, cholesterol ester (Hormal Institute, Austin, Minn.), sphingomyelin, lysolecithin, and lecithin (Lipid Products, South Nutfield, England) was applied to three lanes on both halves of the plate, interspersed at regular intervals with the samples. The concentration of each lipid was approximately $1 \mu \mathrm{g} / \mu \mathrm{l}$. Dry weights of samples and standards were used to ensure that not more than $10 \mu \mathrm{g}$ of a given lipid class was applied to each lane. After development in a solvent system of hexane, diethyl ether, and acetic acid (70:30:1, vol/vol/vol), the bottom $1.5 \mathrm{~cm}$ of silica of the left half of the plate was scraped so that only the right half would be redeveloped in a solvent system of chloroform, methanol, water, and acetic acid $(65: 25: 4: 1)$. Cholesterol, fatty acid, triglyceride, and cholesterol ester were separated on the left, and lysolecithin, sphingomyelin, and lecithin on the right. The plate was then sprayed with $50 \%$ sulfuric acid and heated to $220^{\circ} \mathrm{C}$ for $45 \mathrm{~min}$. The charred lipid spots were scanned with a photodensitometer (Photovolt Corp., New York), and the area under the peaks was measured. For less than $10 \mu \mathrm{g}$ of lipid, the amount of lipid was linearly related to the area. The method was accurate to $\pm 5 \%$ and sufficiently sensitive to quantitate $0.2 \mu \mathrm{g}$ of a given lipid class.

Gas-liquid chromatography. Fatty acids of cholesterol esters were determined using a Hewlett-Packard model 700 gas chromatograph (Hewlett-Packard Corp., Palo Alto, Calif.) equipped with a hydrogen flame ionization detector. A 6-foot column packed with $10 \%$ SP2340 on $100 / 120$ Supelcoport (Supelco Inc., Bellefonte, $\mathrm{Pa}$.) was operated at a temperature of $195^{\circ} \mathrm{C}$. Cholesterol esters were isolated by preparative thin-layer chromatography using a hexane, ether $(94: 6, \mathrm{vol} / \mathrm{vol})$ solvent system. Plates were sprayed with 2,7-dichlorofluorescein (Applied Science Labs, Inc., State College, $\mathrm{Pa}$.), and lipid spats were visualized under ultraviolet light. The cholesterol ester spots were scraped, and the silica was removed by eluting the lipid with chloroform: methanol $(2: 1)$ through a sintered glass filter. The esters were hydrolyzed in $2 \%$ alcoholic $\mathrm{KOH}$ according to the method of Albrink (28) as modified by Smith and Slater (7), except that the initial reaction was allowed to proceed under nitrogen overnight at room temperature before the reactants were heated to $80^{\circ} \mathrm{C}$ for $30 \mathrm{~min}$. Methylation of the fatty acids was accomplished by refluxing at $80^{\circ} \mathrm{C}$ with $14 \% \mathrm{BF}_{3}$ in methanol for $30 \mathrm{~min}$ (29). Methyl esters were extracted into hexane, dried under nitrogen, and redissolved in $0.25 \mathrm{ml}$ hexane. A known mixture of methyl esters (Supelco Inc.) was used to standardize retention times. The relative amounts of methyl esters were determined by the method of multiplying peak height by retention time $(30)$.

\section{Physical methods}

Polarizing microscopy. A Zeiss standard NL microscope (Carl Zeiss, Inc., New York) fitted with a heating and cooling stage was used to identify physical states of lipids in plaques $(2,3)$. Perturbing the coverslip enabled us to observe flow in isotropic liquids and birefringent liquid crystals. A quartz wedge plate was used to determine the optical sign of birefringence. The initial observations were carried out at $23^{\circ} \mathrm{C}$. Thereafter the temperature was increased, and the melting points of liquid crystals and crystals were determined to within $\pm 0.5^{\circ} \mathrm{C}$.

$X$-ray diffraction. X-ray diffraction studies were performed using an Elliot toroidal camera (Baird and Tatlock, London, England) and nickel-filtered $\mathrm{CuK} \alpha$ radiation from an Elliot GX6 rotating anode generator. During exposure, samples of fresh minced plaque were positioned between Mylar windows in a controlled temperature sample holder. $\mathrm{X}$-ray diffraction patterns of cholesterol monohydrate were obtained from cholesterol which had been recrystallized from $30 \%$ aqueous ethanol and extensively washed with distilled water. Anhydrous cholesterol was prepared by recrystallization from absolute ethanol, or by melting and cooling cholesterol monohydrate.

\section{RESULTS}

The chemical and physical data pertaining to ordinary fatty streaks, intermediate lesions, fibrous plaques, and gruel plaques are summarized in Tables I and II, and the relative composition in terms of cholesterol, cholesterol ester, and total phospholipids is illustrated in Fig. 3. Ordinary fatty streaks fall within the two-phase zone, while all the other lesions have compositions in the 
TABLE I

Polarizing Microscopic Findings and Average Lipid Composition Weight Percentages for Each Class of Atherosclerotic Lesion

\begin{tabular}{|c|c|c|c|c|c|c|c|c|c|c|c|}
\hline & Crystals & $\begin{array}{l}\text { Bire- } \\
\text { fringent } \\
\text { droplets }\end{array}$ & $\begin{array}{c}\text { Choles- } \\
\text { terol } \\
(a)\end{array}$ & $\begin{array}{l}\text { Triglyc- } \\
\text { eride } \\
\text { (b) }\end{array}$ & $\begin{array}{c}\text { Choles- } \\
\text { terol } \\
\text { ester } \\
(c)\end{array}$ & $\begin{array}{l}\text { Lyso- } \\
\text { lecithin } \\
(d)\end{array}$ & $\begin{array}{l}\text { Sphingo- } \\
\text { myelin } \\
(e)\end{array}$ & $\begin{array}{c}\text { Lecithin } \\
(f)\end{array}$ & $\begin{array}{l}\text { Total } \\
\text { phospho- } \\
\text { lipid } \ddagger \\
(g)\end{array}$ & $\begin{array}{l}\text { Sphingo- } \\
\text { myelin/ } \\
\text { lecithin\& } \\
\text { (h) }\end{array}$ & $\begin{array}{c}\text { Cholesteryl } \\
\text { oleate/ } \\
\text { cholesteryl } \\
\text { linoleateई } \\
\text { (i) }\end{array}$ \\
\hline Fatty streak (13)* & $2 / 13$ & $13 / 13$ & $9.6 \pm 0.7$ & $2.8 \pm 0.5$ & $77.0 \pm 2.0$ & $0.3 \pm 0.1$ & $5.6 \pm 0.8$ & $4.8 \pm 0.4$ & $10.1 \pm 1.0$ & $1.2 \pm 0.2$ & $1.9 \pm 0.3$ \\
\hline Intermediate lesion (31) & $10 / 31$ & $28 / 31$ & $21.1 \pm 0.6$ & $4.4 \pm 0.5$ & $55.0 \pm 1.5$ & $1.0 \pm 0.2$ & $11.0 \pm 0.7$ & $7.6 \pm 0.6$ & $19.6 \pm 1.0$ & $2.2 \pm 0.5$ & $1.4 \pm 0.1$ \\
\hline Fibrous plaque (27) & $27 / 27$ & $2 / 27$ & $22.5 \pm 1.2$ & $5.2 \pm 0.6$ & $55.5 \pm 1.5$ & $0.6 \pm 0.1$ & $11.7 \pm 0.5$ & $4.5 \pm 0.5$ & $16.8 \pm 0.8$ & $3.5 \pm 0.5$ & $0.8 \pm 0.1$ \\
\hline Gruel plaque (24) & $24 / 24$ & $1 / 24$ & $31.5 \pm 1.9$ & $6.0 \pm 0.7$ & $47.2 \pm 2.3$ & $0.9 \pm 0.2$ & $10.1 \pm 0.5$ & $4.3 \pm 0.4$ & $15.3 \pm 0.7$ & $3.4 \pm 0.7$ & $1.1 \pm 0.1$ \\
\hline
\end{tabular}

$a-i$, statistically significant differences between groups, $P<0.05$, determined by Newman-Keuls test (35).

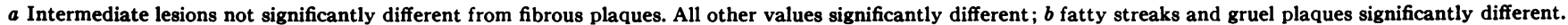

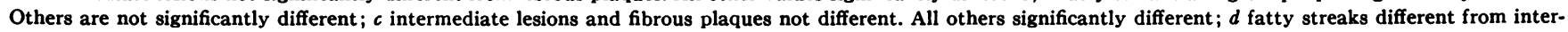

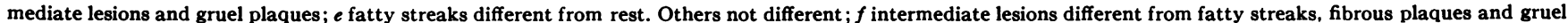

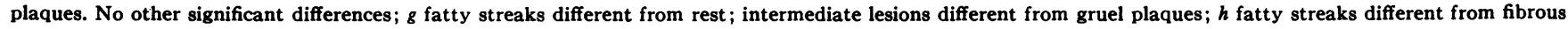
plaques and gruel plaques; $i$ fatty streaks different from fibrous plaques and gruel plaques; intermediate lesions different from fibrous plaques.

* Number of lesions in each group in parentheses.

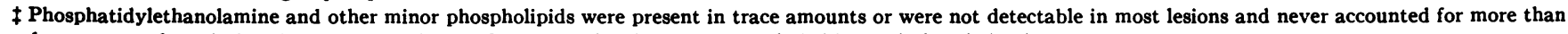
a few percent of total phospholipid in any lesion. Consequently, they are not included in total phospholipids.

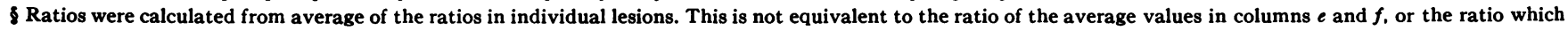
could be calculated from the average values of $C_{18: 1}$ and $C_{18: 2}$ given in Table II.

three-phase zone. Although intermediate lesions and fibrous plaques are indistinguishable by relative compositions of cholesterol, cholesterol ester, and phospholipid, chemical and physical differences between these groups were found and are detailed below.

Fatty streaks. Lipid composition of all 44 lesions classified by appearance under the dissecting microscope as fatty streaks are represented by the points in Fig. 4. 13 fatty streaks have compositions which fall on or below the line and are designated ordinary fatty streaks, while 31 fall above the line and are designated intermediate lesions.

Ordinary fatty streaks. These lesions have significantly less free cholesterol and sphingomyelin and more cholesterol ester than the other types of lesions examined (Table I). They have a lower sphingomyelin/lecithin ratio and a higher cholesteryl oleate/cholesteryl linoleate ratio than advanced plaques. Although they have less triglyceride than other lesions, the difference reaches statistical significance only with gruel plaques.

Much of the accumulated lipid in fatty streaks is present as droplets which are composed mainly of cholesterol esters with small amounts of free cholesterol, triglyceride, and phospholipid $(18,31)$. Fatty streaks have relatively more cholesteryl oleate, and less cholesteryl linoleate and cholesteryl arachidonate than advanced plaques (Table II). This is reflected in all fatty streaks examined having a preponderance of anisotropic droplets at $23^{\circ} \mathrm{C}$ (Fig. 5A) since monoenoic cholesterol esters have higher melting points than polyunsaturated esters (2). The optical sign of birefringence of the droplets was invariably positive, indicating a smectic liquid crystalline state (32). By microscopic observation they do not coalesce when they come into contact, implying that either their surface is stabilized with a phospholipid monolayer or they are membrane-bound. On heating, most of the anisotropic droplets melt by $37^{\circ} \mathrm{C}$, and nearly all are in an isotropic liquid state at about $45^{\circ} \mathrm{C}$ (Fig. 5B). ${ }^{1}$ Thus, at body temperature the majority of

${ }^{1}$ Infrequently, droplets are seen which retain a positive birefringence to $100^{\circ} \mathrm{C}$. Since all biologically important cholesteryl esters melt to an isotropic state well below $100^{\circ} \mathrm{C}$

TABLE II

Weight Percentages of Cholesterol Ester Fatty Acids

\begin{tabular}{|c|c|c|c|c|c|c|c|c|c|c|}
\hline & $\mathbf{C}_{14}$ & $C_{16: 0}$ & $C_{16: 1}$ & $C_{18: 0}$ & $C_{18: 1}$ & $C_{18: 2}$ & $C_{20: 1}$ & $C_{20: 2}$ & $C_{20: 2}$ & $C_{20: 4}$ \\
\hline Fatty streaks $(11)^{*}$ & $1.7 \pm 0.3$ & $13.0 \pm 1.7$ & $4.6 \pm 0.8$ & $6.2 \pm 2.7$ & $34.6 \pm 3.4$ & $26.1 \pm 4.4$ & $1.7 \pm 0.7$ & $1.0 \pm 0.3$ & $0.9 \pm 0.3$ & $7.5 \pm 0.8$ \\
\hline Intermediate lesions (30) & $1.7 \pm 0.3$ & $13.1 \pm 0.7$ & $4.8 \pm 0.3$ & $3.1 \pm 0.6$ & $35.5 \pm 1.5$ & $30.3 \pm 1.9$ & $0.8 \pm 0.3$ & $0.4 \pm 0.1$ & $1.1 \pm 0.3$ & $7.6 \pm 0.3$ \\
\hline Fibrous plaques (26) & $1.1 \pm 0.3$ & $12.2 \pm 0.4$ & $5.3 \pm 0.4$ & $2.0 \pm 0.3$ & $28.3 \pm 1.2$ & $37.2 \pm 1.4$ & $0.5 \pm 0.2$ & $0.6 \pm 0.1$ & $0.8 \pm 0.2$ & $10.6 \pm 0.6$ \\
\hline Gruel plaques (19) & $0.7 \pm 0.1$ & $11.0 \pm 0.8$ & $4.5 \pm 0.3$ & $2.0 \pm 0.3$ & $32.3 \pm 1.9$ & $34.9 \pm 2.0$ & $0.6 \pm 0.1$ & $0.9 \pm 0.2$ & $1.0 \pm 0.3$ & $10.2 \pm 0.7$ \\
\hline
\end{tabular}

Statistically significant differences between groups, $P<0.05$, determined by Newman-Keuls test (35).

$\mathrm{C}_{\text {18:0 }}$ Fatty streaks different from fibrous plaques and gruel plaques. Fatty streaks not different from intermediate lesions; $\mathrm{C}_{18: 1}$ Intermediate lesions different from fibrous plaques; $C_{18: 2}$ Fatty streaks different from fibrous plaques and gruel plaques; $C_{20: 1}$ Fatty streaks different from fibrous plaques; $C_{20: 4}$ Fatty streaks different from fibrous plaques and gruel plaques. Intermediate lesions different from fibrous plaques and gruel plaques.

* Number of lesions in each group in parentheses. 


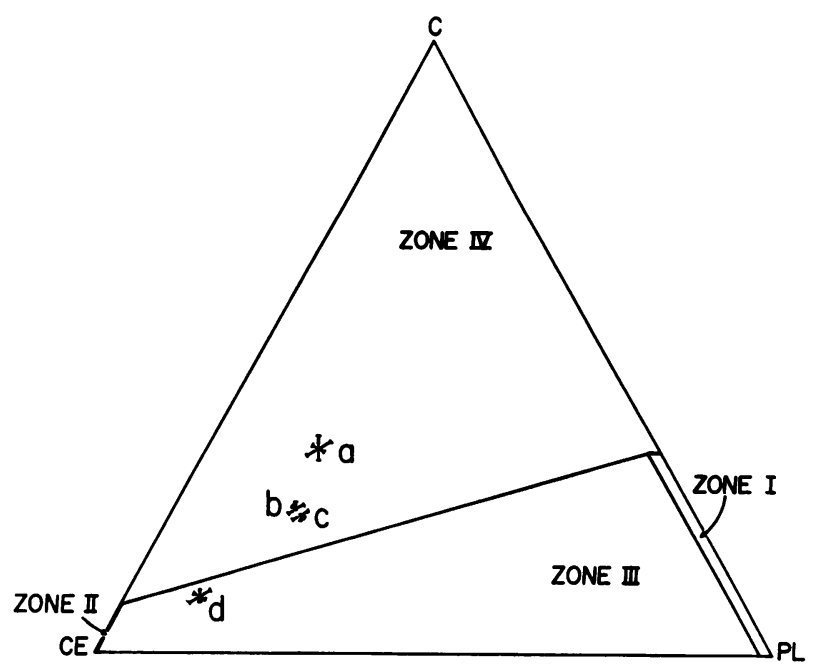

Figure 3 Mean composition of 24 individual gruel plaques $(a), 27$ fibrous plaques $(b), 31$ intermediate lesions $(c)$, and 13 fatty streaks $(d)$. Bars represent \pm SEM.

lipid droplets are in the liquid state, and a minority are in the smectic liquid crystalline state. This latter state is thermotropically stable since cooling to $4^{\circ} \mathrm{C}$ does not precipitate crystals of cholesterol ester. A very rare cholesterol monohydrate crystal was seen in only 2 of the 13 ordinary fatty streaks.

$\mathrm{X}$-ray diffraction studies of fatty streaks at $23^{\circ} \mathrm{C}$ showed a sharp diffraction line at $35 \AA$ and a diffuse band at $5 \AA$ (Fig. $6 a$ ). Heating to $40-45^{\circ} \mathrm{C}$ abolished the $35 \AA$ line (Fig. $6 b$ ), but after cooling to $23^{\circ} \mathrm{C}$ this diffraction line was again present. The diffraction pattern at $23^{\circ} \mathrm{C}$ (Fig. 6 c) was identical to that of a mixture of cholesteryl oleate and cholesteryl linoleate $(6 / 4 \mathrm{~mol} /$ $\mathrm{mol}$ ) in the smectic liquid crystalline state (Fig. 6d). After drying, fatty streaks show a crystalline diffraction pattern at $23^{\circ} \mathrm{C}$, probably produced by crystallization or co-crystallization of several cholesterol esters. This correlated with our observations by polarizing microscopy that crystals form within lipid droplets on prolonged drying. Water does not interact with these crystals; however, on heating, they melt to an isotropic liquid at $45-50^{\circ} \mathrm{C}$, and with subsequent cooling smectic liquid crystals form. This behavior is typical of mixtures of unsaturated cholesterol esters (2).

Intermediate lesions. The points above the line in Fig. 4 represent the 31 intermediate lesions. Table I shows that these lesions have values intermediate between fatty streaks and advanced plaques with respect

(2) and since lecithin-water liquid crystals do not melt until well above $100^{\circ} \mathrm{C}(3)$, we conclude that the rare droplets which remain anisotropic to $100^{\circ} \mathrm{C}$ are phospholipid-rich lamellar liquid crystals analogous to those which would be found in zone I of Fig. 1. The lamellar structure of this type of droplet has been demonstrated in atheroscleratic lesions by electron microscopy $(13,33)$. to cholesterol, triglyceride, cholesterol ester, sphingomyelin/lecithin ratio, cholesteryl oleate/cholesteryl linoleate ratio, and numbers of lesions with cholesterol crystals and anisotropic lipid droplets. While the relative amounts of most individual cholesterol esters are the same in fatty streaks and intermediate lesions, Table II shows that values for two cholesterol esters, cholesteryl stearate and linoleate, are intermediate between fatty streaks and advanced lesions. Intermediate lesions differed significantly from both fatty streaks and gruel plaques with respect to relative content of cholesterol, cholesterol ester, lecithin, and total phospholipid (Table I). They also differed significantly from fatty streaks in their content of sphingomyelin. Although similar to fibrous plaques with respect to cholesterol, cholesterol ester, and total phospholipid (Table I), they are significantly different in their relative amounts of lecithin, their cholesteryl oleate/cholesteryl linoleate ratio, and relative content of cholesteryl oleate and arachidonate (Table II).

Like fatty streaks, most (28/31) intermediate lesions had a preponderance of anisotropic droplets at $23^{\circ} \mathrm{C}$. However, 10 lesions had cholesterol monohydrate crystals, a feature characteristic of advanced plaques, but not of ordinary fatty streaks. 21 intermediate lesions had no observable crystals, and yet their chemical composition placed them in the three-phase region of the phase diagram, indicating that there may be a stage of atherosclerosis when the phospholipid or droplet phase, or both, are supersaturated with respect to cholesterol.

Fibrous plaques. The compositions of 27 fibrous plaques are shown in Fig. 7. All but one are in the three-phase zone (that is, above the line), and all had

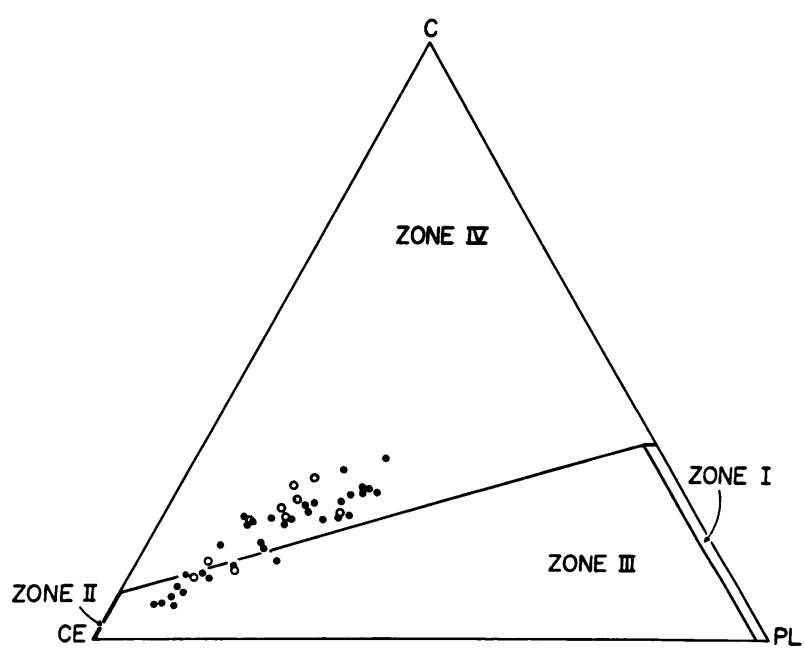

FIGURE 4 Relative composition of individual ordinary fatty streaks below the line, and intermediate lesions above the line. Lesions with crystals $(O)$, and lesions without crystals (๑). 

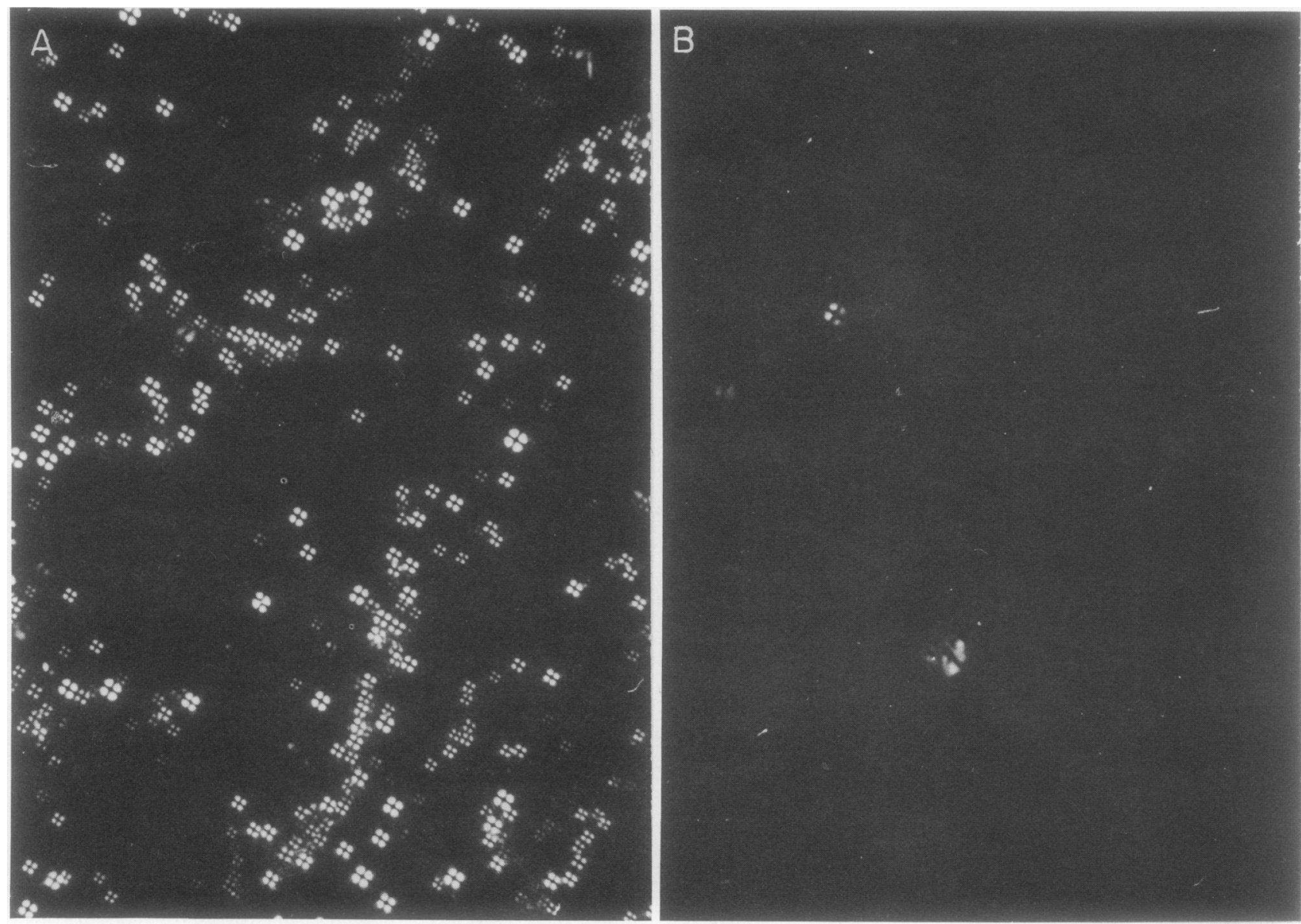

FigURE 5 Anisotropic droplets of a fatty streak ( $\times 400$, crossed polarizers). (A) appearance at $23^{\circ} \mathrm{C}$, and (B) appearance at $45^{\circ} \mathrm{C}$.

cholesterol crystals by microscopy. Fig. 3 shows that the average composition of fibrous plaques is generally lower in the phase diagram than gruel plaques, implying that they had smaller amounts of excess free cholesterol. This correlated well with our microscopic findings that fibrous plaques had fewer crystals than gruel plaques. The large percentage of the polyunsaturated cholesterol esters, cholesteryl linoleate, and cholesteryl arachidonate (Table II) correlated with the fact that most lipid droplets in fibrous plaques are isotropic at $23^{\circ} \mathrm{C}$, since unsaturation decreases the melting point of cholesterol ester (2).

Gruel plaques. The composition of each of the 24 gruel plaques is plotted in Fig. 7. A relatively large proportion of the lipids in gruel plaques is free cholesterol (Table I). If a line were drawn from the cholesterol apex through each point representing a gruel plaque to the line separating the two-phase zone from the threephase zone, the distance from the point to that line would be proportional to the excess free cholesterol. Under equilibrium conditions, this excess free cholesterol should be present as crystals. Polarizing light microscopy of gruel plaques revealed a profusion of cholesterol monohydrate crystals often clustered and stacked one upon the other (Fig. 8). The morphology of the crystals of the plaque is very similar to the cholesterol monohydrate crystals of bile, being rhomboidal plates with interfacial (corner) angles of $79-80^{\circ}$.

To confirm the chemical identity of the plate crystals, $\mathrm{X}$-ray diffraction studies were performed on nine gruel plaques from nine arterial specimens. The same diffraction pattern was obtained from all samples and remained unchanged on heating the sample to $38^{\circ} \mathrm{C}$. The diffraction spacings and intensity distribution of both gruel plaques and their isolated crystals were identical to pure cholesterol monohydrate crystals (Table III; Fig. 9). Two plaques were exposed to air for several days, and the diffraction patterns changed to one showing diffraction lines from both cholesterol monohydrate and anhydrous cholesterol, thus emphasizing the importance of studying fresh tissue (21).

Gruel plaques have less cholesterol ester than other 


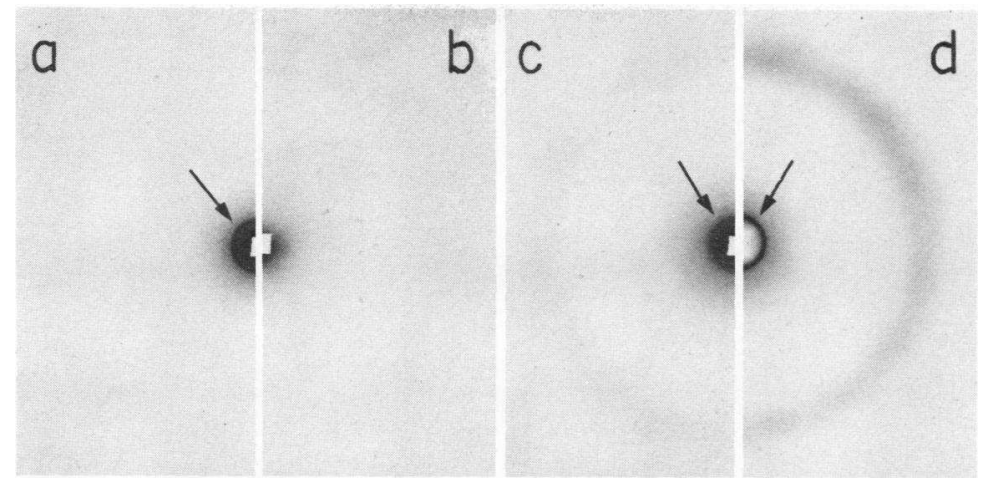

\begin{abstract}
FIGURE $6 \mathrm{X}$-ray diffraction pattern of fatty streaks and cholesterol esters: (a) X-ray diffraction pattern of a fatty streak at $23^{\circ} \mathrm{C}$ and (b) at $45^{\circ} \mathrm{C}$. The $35 \AA$ line has disappeared at $45^{\circ} \mathrm{C}$. (c) X-ray diffraction pattern of a fatty streak showing both the $35 \AA$ line and the $5 \AA$ band, compared with (d) cholesteryl oleate/cholesteryl linoleate (mole ratio 6:4) in the smectic liquid crystalline state at $23^{\circ} \mathrm{C}$. The physical state of the cholesterol ester mixture was verified by polarizing microscopy. The single low-angle diffraction maximum at $35 \AA$ (arrow) is characteristic of the smectic phase of mono- and di-unsaturated C-18 fatty esters of cholesterol, mixtures of these esters, or more heterogeneous mixtures of cholesterol esters such as those in fatty streaks $(a, c)$, and low-density lipoproteins and their isolated esters (34).
\end{abstract}

lesions (Table I), and a relatively large proportion of the fatty acids of the cholesterol esters are polyunsaturated (Table II). Therefore, as in fibrous plaques, most of the lipid droplets are isotropic at $23^{\circ} \mathrm{C}$.

The Spearman rank correlation test (35) was used to determine whether ranking lesions by groups in the order of (a) fatty streaks, (b) intermediate lesions, (c) fibrous plaques, and $(d)$ gruel plaques would correlate with a progressive increase or decrease for each chemical or physical determination. Strong positive or negative correlations are indicated by a high-rank correlation coefficient $\left(r_{s}\right) .14$ of the 21 rank correlations were statistically significant. Strong correlations were obtained for cholesterol crystals $r_{0}=0.82 \quad(P<0.001)$, birefringent droplets $r_{t}=0.81(P<0.001)$, cholesterol $r_{t}=$ $0.66(P<0.001)$, and cholesterol ester $r_{s}=-0.54(P<$ $0.001)$. Weaker correlations were triglyceride $r_{t}=0.36$ $(P<0.001)$, sphingomyelin/lecithin ratio $r_{\bullet}=0.43(P<$ 0.001 ), cholesteryl oleate/cholesteryl linoleate ratio $r_{s}=$ $-0.35(P<0.001)$, cholesteryl myristate $r_{t}=-0.38$ $(P<0.001)$, cholesteryl arachidonate $r_{\bullet}=0.45 \quad(P<$ $0.001)$, lecithin $r_{0}=-0.26(P<0.01)$, sphingomyelin $r_{t}=0.25(P<0.02)$, cholesteryl linoleate $r_{t}=0.30(P<$ $0.006)$, and cholesteryl oleate $r_{\mathrm{t}}=-0.19 \quad(P<0.08)$. The correlation coefficient for total phospholipids and for the other individual cholesterol esters were not statistically significant.

\section{DISCUSSION}

Most previous studies of the lipids of atherosclerotic plaques have not concerned themselves with the physical state of the lipids. However, in our study of 95 individual atherosclerotic lesions, we found that lipids of fatty streaks exist in two states, liquid and liquid crystalline, with a third state, crystalline, being present in advanced plaques. The constituents of these states are $(a)$ cholesterol ester-rich droplets which may be either liquid or liquid crystalline at $37^{\circ} \mathrm{C},(b)$ bilayer arrangements of phospholipid liquid crystals in membranes and myelin figure-like deposits, and (c) cholesterol monohydrate crystals. Although cholesterol esters and phospholipids may both be liquid crystals, they are largely immiscible in each other and thus form separate phases $(5,36)$.

The explanation of the phase behavior of the major plaque lipids is provided by the phase diagram of free cholesterol, phospholipid, and cholesterol ester in excess

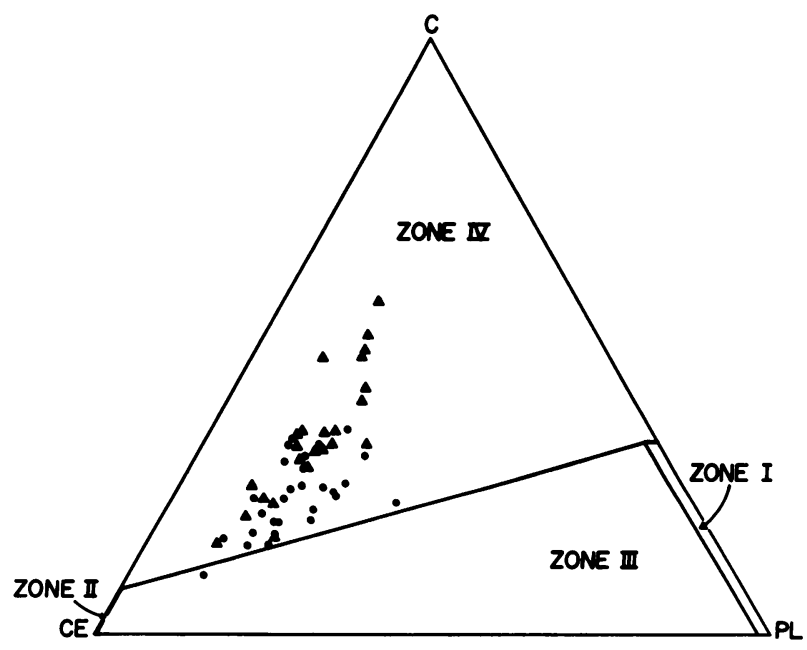

Figure 7 Relative lipid composition of 27 fibrous plaques (•) and 24 gruel plaques ( $\Delta$ ). 


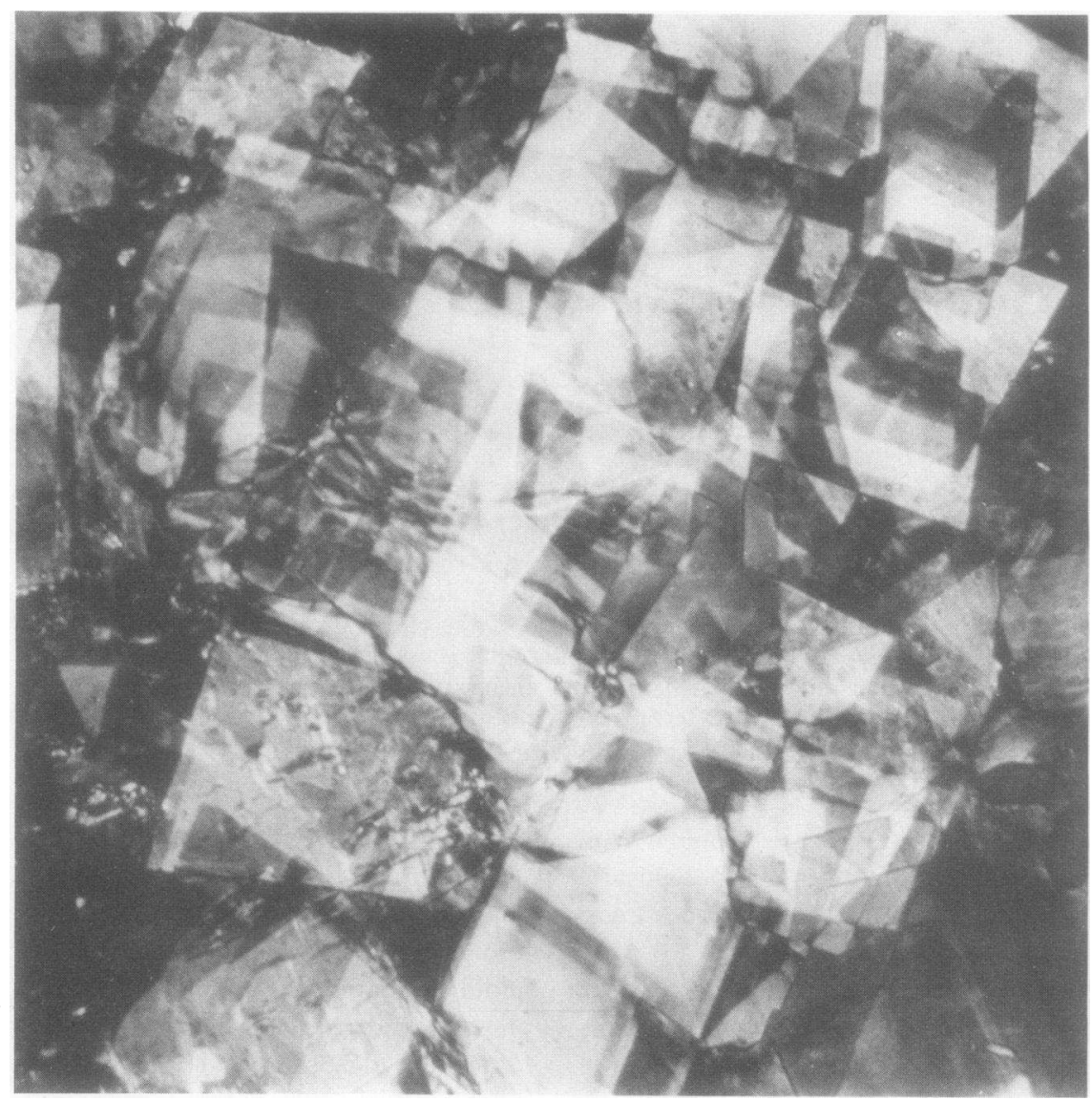

Figure 8 Cholesterol monohydrate plate crystals from plaques at $25^{\circ} \mathrm{C}(\times 100$, crossed polarizers).

water (Fig. 1). This diagram predicts that phospholipid liquid crystals (e.g., membranes) become saturated with small amounts $(<2 \%)$ of cholesterol ester, and any additional cholesterol ester must form a second phase. Free cholesterol, which is virtually insoluble in water, can be solubilized by the phospholipid phase up to $33 \%$ (wt/wt) and by the cholesterol ester phase up to about $8 \%$ $(w t / w t) .^{2}$ When cholesterol is present in greater concentrations, the system must either be supersaturated (nonequilibrium conditions) or contain cholesterol crystals (equilibrium conditions).

The applicability of the model system to the lipids of human atherosclerotic plaques has been demonstrated in our study. 50 advanced plaques had a chemical composition which placed them in the three-phase zone, and cholesterol monohydrate crystals and cholesterol ester

\footnotetext{
The absolute amount of cholesterol incorporated into cholesterol ester varies from as low as $4 \%$ for pure cholesteryl oleate to $8 \%$ for pure cholesteryl linoleate and for mixtures of esters rich in cholesteryl linoleate or other polyunsaturated esters of cholesterol (D. M. Small, unpublished observations).
}

droplets were demonstrated in all. (The phospholipid liquid crystalline phase may reasonably be assumed to be present, since membranes are a constituent of plaques. see references 13 and 14.). Only one advanced plaque with crystals did not lie in the three-phase zone. Of 13 fatty streaks in the two-phase zone, 11 were shown to have only two phases, since no observable crystals were present. Two fatty streaks which were near the line delineating the three-phase zone had a small number of cholesterol monohydrate crystals. Thus, there was good agreement between the simplified in vitro model system and the more complex lipid system of the plaque. Since only lipid-lipid interactions are considered in the phase diagram, lipid-protein interactions, while undoubtedly present, must have a relatively small influence on lipid physical states. Moreover, since some of the lipids in the lesions we have studied were likely to be intracellular, especially in fatty streak lesions, phase equilibrium considerations are applicable to lipid accumulations whether they are intra- or extracellular.

Despite the good correlation between the in vitro 
model system and the lipid systems of atherosclerotic lesions, there are some reservations to applying phase equilibrium considerations to the lipids of the whole lesion. A model system is defined by the contents of a test tube. That is, each lipid system has a fixed chemical composition and limited boundaries. At a given temperature and pressure, the components of this system reach thermodynamic equilibrium after an adequate period of mixing. In the atherosclerotic lesion an analogous system would exist if the lipid composition remained constant within the limits of the lesion and the lipid molecules could freely diffuse from one region of the lesion to another and reach equilibrium. However, if lipids are compartmentalized, for instance in a region of a lesion or even in a cell, then it is possible that the chemical composition will vary from one compartment to another. The physical state of the lipids in such compartments will be determined not only by the chemical composition but also by the rate with which the chemical composition changes either due to metabolic processes or transfer of lipids from one compartment to another. In fact, the data of Smith and Slater (7) when plotted on the phase diagram show that relative chemical compositions differ in different regions of advanced plaques (5). Furthermore, the small droplets which make up the oily cholesterol ester phase melt at different temperatures, indicating they must have a slightly different chemical composition. Thus, our physicochemical approach to the lipids of the

TABLE III

$X$-Ray Diffraction Spacings and Intensity Distributions of Cholesterol Monohydrate and Gruel Plaques

\begin{tabular}{|c|c|c|}
\hline \multirow{2}{*}{$\begin{array}{l}\text { Interplanar } \\
\text { spacing }\end{array}$} & \multicolumn{2}{|c|}{ Relative intensity } \\
\hline & Cholesterol monohydrate & Gruel plaques \\
\hline \multicolumn{3}{|l|}{$d(\AA)$} \\
\hline 33.5 & Strong & Strong \\
\hline 16.8 & Moderately strong & Moderately strong \\
\hline 11.7 & Weak & - \\
\hline 11.4 & - & Weak* \\
\hline 5.9 & Strong & Moderately strong \\
\hline 5.7 & - & Weak \\
\hline 5.5 & Weak & Weak \\
\hline 5.3 & Weak & - \\
\hline 5.1 & - & Weak* \\
\hline 4.9 & Moderately weak & Moderately weak \\
\hline 4.7 & Moderately weak & Weak \\
\hline 4.5 & Very weak & - \\
\hline 4.3 & Very weak & Weak \\
\hline 3.8 & Moderate & Moderate \\
\hline 3.5 & Very weak & Very weak \\
\hline
\end{tabular}

* Diffraction line from plaque specimen is difficult to measure because of weak intensity and granularity. Diffraction spacing is probably identical with slightly larger spacing of pure cholesterol monohydrate.

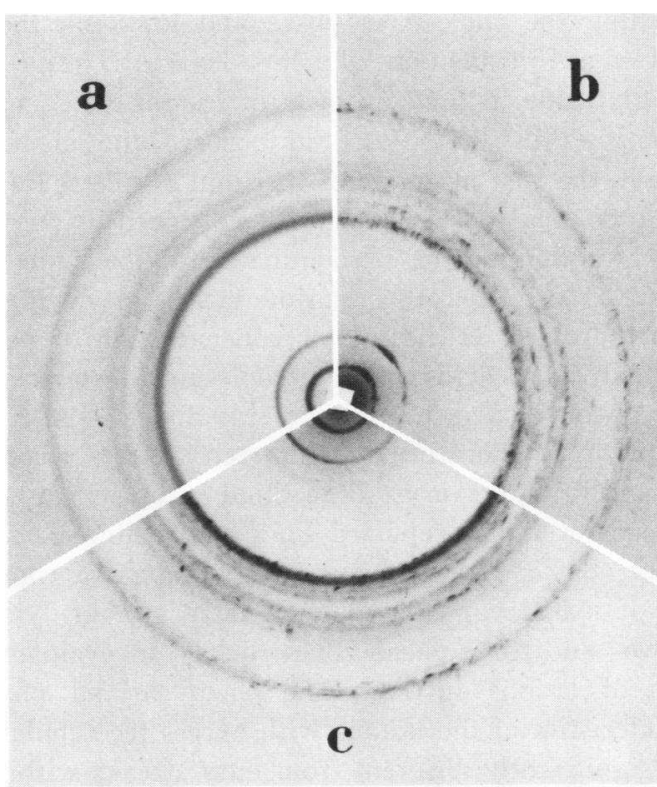

FIGURE 9 Composite X-ray diffraction pattern at $20^{\circ} \mathrm{C}$ of (a) cholesterol monohydrate, (b) a gruel plaque, and (c) isolated crystals from a gruel plaque.

whole lesion must be considered a first approximation in that it gives the mean compostion of the individual lesion and predicts the general physical states in which the lipids should exist at equilibrium. It may mask small compartments differing from the mean composition. Nevertheless, even though some compartmentalization may occur within a lesion, substantial anomalies in the predicted physical states do not occur except in some of the intermediate lesions.

The relatively small concentrations of triglycerides in atherosclerotic lesions (less than $10 \%$ ) can be expected to have minor effects on the physical state of the lipids. Most of the triglyceride appears to be present in the cholesterol ester-rich droplets (18-19). Model systems of triolein and cholesteryl oleate show that these lipids form simple eutectic systems in which complete mutual solubility exists above the melting points of both compounds (2). Further, small amounts of triglyceride (2\%) added to mixed cholesterol esters will abolish the cholesteric phase, and slightly more triglyceride (about $5 \%$ ) will lower the isotropic-smectic transition temperature by about $5^{\circ} \mathrm{C}$ (R. Deckelbaum and D. M. Small, unpublished observations). Since the solubility of the cholesterol in cholesterol ester is not enhanced by the presence of triglycerides (2), it is unlikely that the phase equilibrium of cholesterol in the model system would be significantly affected by the presence of small amounts of triglyceride. Thus, small amounts of triglyceride should partition mainly into the cholesterol ester phase, causing little changes in the solubility of cholesterol, but 
abolishing the cholesteric phase and lowering the isotropic-smectic transition by a few degrees. The presence of triglyceride in lipid droplets of lesions (19) may be responsible for the failure to observe the cholesteric phase in the vast majority of the lipid droplets. Further, the higher triglyceride content of advanced plaques may partly account for the predominance of isotropic droplets at $23^{\circ} \mathrm{C}$. The other major factor producing low melting droplets is the higher concentration of polyunsaturated fatty acids of the cholesterol esters. Fatty streaks which have less triglyceride and a smaller proportion of polyunsaturated cholesterol esters have predominantly liquid crystalline lipid droplets at $23^{\circ} \mathrm{C}$.

The diversity of reported lipid compositions of fatty streaks $(8,23,24)$, and our own findings of compositions of macroscopically defined fatty streaks lying in the two- and three-phase zones, led us to evaluate this group of lesions further. The chemical and physical characteristics of the lesions with excess free cholesterol were significantly different from fatty streaks within the two-phase zone, being intermediate in many respects between fatty streaks and advanced plaques. Identification of this group of intermediate lesions lends support to the hypothesis that at least some fatty streaks evolve into advanced plaques. Since the lipid phases of many intermediate lesions appear to be supersaturated with free cholesterol, a metastable condition probably preceeds the deposition of crystalline cholesterol in plaques.

Lipid accumulation in atherosclerosis must be associated with a slower net rate of efflux than influx of lipids in the vessel wall. Since the mobility of molecules in crystals, and to a lesser extent in liquid crystals, is restricted (37), lipids existing in these physical states would have a slower rate of turnover, and would thus be localized in a relatively inert metabolic pool $(38,39)$. Moreover, the organization of lipid molecules in crystals and liquid crystals may well limit enzyme accessibility either to the substrate molecules themselves or to specific substrate sites within molecules. Thus, physical states of lipid would be of importance both in formation and regression of plaques.

\section{ACKNOWLEDGMENTS}

We are grateful to Drs. D. T. Downing and E. L. Rogers for helpful discussions, Dr. R. C. Darling of the Massachusetts General Hospital for providing the surgical specimens, and Dr. H. Kayne and Ms. Linda Rose for assistance with statistical analysis. We thank Ms. Joan Fasulo for excellent technical assistance, and Ms. Susan Wydra and Mr. John Steiner for help in preparation of the manuscript.

This work was supported by U. S. Public Health Service grants AM 11453 and HL 18623 (Dr. D. M. Small, Principal Investigator).

\section{REFERENCES}

1. Small, D. M., and M. Bourges. 1966. Lyotropic paracrystalline phases obtained with ternary and quarter- nary systems of amphiphilic substances in water: Studies on aqueous systems of lecithin, bile salt and cholesterol. Mol. Cryst. 1 : 541-561.

2. Small, D. M. 1970 . The physical state of lipids of biolagical importance: Cholesteryl esters, cholesterol, triglyceride. In Surface Chemistry of Biological Systems. M. Blank, editor. Plenum Publishing Corporation, New York. 55-83.

3. Small, D. M. 1967. Phase equilibria and structure of dry and hydrated egg lecithin. J. Lipid Res. 8: 551-557.

4. Shipley, G. G., L. S. Avecilla, and D. M. Small. 1974. Phase behavior and structure of aqueous dispersions of sphingomyelin. J. Lipid Res. 15: 124-131.

5. Small, D. M., and G. G. Shipley. 1974. Physical-chemical basis of lipid deposition in atherosclerosis. The physical state of the lipids helps to explain lipid deposition and lesion reversal in atherosclerosis. Science (Wash. D. C.). 185: 222-229.

6. Buck, R. C., and R. J. Rossiter. 1951. Lipids of normal and atherosclerotic aortas. A chemical study. Arch. Pathol. 51: 224-237.

7. Smith, E. B., and R. S. Slater. 1972. The microdissection of large atherosclerotic plaques to give morphologically and topographically defined fractions for analysis. Part I. The lipids in the isolated fractions. Atherosclerosis. 15 : 37-56.

8. Panganamala, R. V., J. C. Geer, H. M. Sharma, and D. G. Cornwell. 1974. The gross and histologic appearance and the lipid composition of normal intima and lesions from human coronary arteries and aorta. Atherosclerosis. 20: 93-104.

9. Field, H., Jr., L. Swell, P. E. Schools, Jr., and C. R. Treadwell. 1960. Dynamic aspects of cholesterol metabolism in different areas of the aorta and other tissues in man and their relationship to atherosclerosis. Circulation. 22 : 547-558.

10. Dayton, S., S. Hashimoto, and M. L. Pearce. 1965. Influence of a diet high in unsaturated fat upon composition of arterial tissue and atheromata in man. Circulaiton. 32 : 911-924.

11. Luddy, F. E., R. A. Banford, R. W. Riemenschneider, and J. D. Evans. 1958. Fatty acid composition of component lipides from human plasma and atheromas. $J$. Biol. Chem. 232: 843-851.

12. Bottcher, C. J. F., and F. P. Woodford. 1962. Chemical changes in the arterial wall associated with atherosclerosis. Fed. Proc. 21 (Suppl. 2) : 15-19.

13. Ghidoni, J. J., and R. M. O'Neal. 1967. Ultrastructure of human atheroma. Exp. Mol. Pathol. 7: 378-400.

14. Weller, R. O., R. A. Clark, and W. B. Oswald. 1968. Stages in the formation and metabolism of intracellular lipid droplets in atherosclerosis. An electron microscopial and biochemical study. J. Atheroscler. Res. 8: 249-263.

15. Weller, R. O. 1968. A comparison between the intracellular lipid droplets in fatty streaks and those in the more advanced atherosclerotic lesions. Colloq. Int. Cent. Natl. Rech. Sci. 169: 175-185.

16. Stewart, G. T. 1959. Liquid crystals of lipide in normal and atheromatous tissue. Nature (Lond.). 183: 873-875.

17. Hata, Y., and W. Insull, Jr. 1973. Significance of cholesterol esters as liquid crystal in human atherosclerosis. Jpn. Circ. J. 37: 269-275.

18. Lang, P. D., and W. Insull, Jr. 1970. Lipid droplets in atherosclerotic fatty streaks of human aorta. J. Clin. Invest. 49: 1479-1488. 
19. Hata, Y., J. Hower, and W. Insull, Jr. 1974. Cholesteryl ester-rich inclusions from human aortic fatty streak and fibrous plaque lesions of atherosclerosis. I. Crystalline properties, size and internal structure. Am. J. Pathol. 75: $423-456$.

20. Jones, D. B., and P. M. Iannaccone. 1975. Atheromatous emboli in renal biopsies. An ultrastructural study. Am. J. Pathol. 78: 261-276.

21. Bogren, H., and K. Larsson. 1963. An x-ray diffraction study of crystalline cholesterol in some pathological deposits in man. Biochim. Biophys. Acta. 75: 65-69.

22. Lundberg, B., and T. Neovius. 1974. The correlation of physical state and chemical composition of lipids in atherosclerotic lesions. Acta Acad. Aboensis. Ser. B. $34: 1-10$.

23. Insull, W., Jr., and G. E. Bartsch. 1966. Cholesterol, triglyceride, and phospholipid content of intima, media, and atherosclerotic fatty streak in human thoracic aorta. J. Clin. Invest. 45: 513-523.

24. Smith, E. B. 1965. The influence of age and atherosclerosis on the chemistry of aortic intima. Part I. The Lipids. J. Atheroscler. Res. 5: 224-240.

25. Classification of atherosclerotic lesions. Report of a study group. 1958. WHO Tech. Rep. Ser. 143: 3-20.

26. Folch, J., M. Lees, and G. H. Sloane Stanley. 1957. A simple method for isolation and purification of total lipides from animal tissues. J. Biol. Chem. 226: 497-509.

27. Downing, D. T. 1968. Photodensitometry in the thinlayer chromatographic analysis of neutral lipids. $J$. Chromatogr. 38: 91-99.

28. Albrink, M. J. 1959. The microtitration of total fatty acids of serum, with notes on the estimation of triglycerides. J. Lipid Res. 1: 53-59.

29. Metcalfe, L. D., A. A. Schmitz, and J. R. Pelka. 1966. Rapid preparation of fatty acid esters from lipids for gas chromatographic analysis. Anal. Chem. 38: 514-515.
30. Carroll, K. K. 1961. Quantitative estimation of peak areas in Gas-Liquid Chromatography. Nature (Lond.). 191: 377-378.

31. Katz, S. S., D. M. Small, G. G. Shipley, and E. L. Rogers. 1974. Identification and characterization of three lipid phases in human atherosclerotic plaques. Circulation. 50 (Suppl. 3) : 6. (Abstr.)

32. Hartshorne, N. H., and A. Stuart. 1970. Crystals and the Polarizing Microscope. Arnold Ltd., London. 4th edition. 503-555.

33. Haust, M. D. 1968. Electron microscopic and immunohistochemical studies of fatty streaks in human aorta. Prog. Biochem. Pharmacol. 4: 429-437.

34. Deckelbaum, R. J., G. G. Shipley, D. M. Small, R. S. Lees, and P. K. George. 1975. Thermal transitions in human plasma low density lipoproteins. Science (Wash. D. C.). 190: 392-394.

35. Snedecor, G. W., and W. G. Cochran. 1967. Statistical Methods. The Iowa State University Press, Ames. 6th edition.

36. Janiak, M. J., C. R. Loomis, G. G. Shipley, and D. M. Small. 1974. The ternary phase diagram of lecithin, cholesteryl linoleate and water: Phase behavior and structure. J. Mol. Biol. 86: 325-339.

37. Shipley, G. G. 1974. The liquid crystalline behavior of lipids. In Fundamentals of Lipid Chemistry. R. M. Burton and F. G. Guerra, editors. BI-Science Publications Div., Webster Groves, Mo. 2nd edition. 585-612.

38. Chobanian, A. V., and W. Hollander. 1962. Body cholesterol metabolism in man. I. The equilibration of serum and tissue cholesterol. J. Clin. Invest. 41: 1732-1737.

39. Jagannathan, S. N., W. E. Connor, W. H. Baker, and A. K. Bhattacharyya. 1974. The turnover of cholesterol in human atherosclerotic arteries. J. Clin. Invest. 54: 366-377. 